\title{
CÁLCULO DO VOLUME NA EQUAÇÃo DE van der WAALS PELO MÉTODO DE CARDANO
}

Nelson H. T. Lemes

Departamento de Ciências Exatas, Universidade Federal de Alfenas, 37130-000 Alfenas - MG, Brasil

Jessé M. Oliveira e João P. Braga*

Departamento de Química, Instituto de Ciências Exatas, Universidade Federal de Minas Gerais,

31270-901 Belo Horizonte - MG, Brasil

Recebido em 26/10/09; aceito em 3/2/10; publicado na web em 21/5/10

\begin{abstract}
VOLUME CALCULATION IN van der WAALS EQUATION BY THE CARDANO METHOD. Analytical solutions of a cubic equation with real coefficients are established using the Cardano method. The method is first applied to simple third order equation. Calculation of volume in the van der Waals equation of state is afterwards established. These results are exemplified to calculate the volumes below and above critical temperatures. Analytical and numerical values for the compressibility factor are presented as a function of the pressure. As a final example, coexistence volumes in the liquid-vapor equilibrium are calculated. The Cardano approach is very simple to apply, requiring only elementary operations, indicating an attractive method to be used in teaching elementary thermodynamics.
\end{abstract}

Keywords: van der Waals; Cardano; thermodynamic.

\section{INTRODUÇÃO}

O livro A grande arte sobre as regras da álgebra, de 1545, escrito por Girolamo Cardano (1501-1576), ${ }^{1}$ contém argumentos geométricos usados para obtenção das soluções da equação do terceiro e quarto graus. A versão traduzida para o inglês, de 1968, contém, no prefácio, uma discussão sobre os aspectos históricos envolvendo a solução de Cardano. Esses aspectos históricos não serão discutidos aqui, mas podem ser encontrados também na referência 2. Trechos de outros artigos sobre a história da álgebra estão compilados na referência 3 .

A teoria e aplicações da solução de Cardano para equações completas do terceiro grau, $x^{3}+b x^{2}+c x+d=0$, serão aqui exploradas. Inicialmente a teoria para se chegar às três soluções possíveis será apresentada, restringindo o problema a soluções reais. Exemplos simples e tomados do livro de Cardano serão dados para ilustrar a teoria. Na parte final do artigo aplicações são feitas para sistemas descritos pela equação de van der Waals.

O desenvolvimento do presente trabalho foi motivado pelo problema de se calcular o volume na equação de van der Waals. Para um dado sistema, o cálculo desse volume consiste na solução de uma equação cúbica. ${ }^{4}$ A raiz maior pode ser usada, para o cálculo do fator de compressibilidade no sistema gasoso. As raizes extremas podem ser usadas para descrever o equílíbrio líquido-vapor, como será ilustrado. Os resultados aqui apresentados são gerais e podem ser usados em outras áreas da quimica, como em equilíbrio químico. Essas aplicações serão feitas posteriormente.

\section{EQUAÇÃO EQUIVALENTE}

O objetivo inicial será o de resolver uma equação algébrica do terceiro grau,

$$
x^{3}+b x^{2}+c x+d=0
$$

com coeficientes reais. O primeiro passo é tentar a substituição, 5. $x=y+\alpha$, fornecendo, $y^{3}+y^{2}(3 \alpha+b)+y\left(c+3 \alpha^{2}+2 b \alpha\right)+\left(\alpha^{3}+b \alpha^{2}+c \alpha+d\right)=0$

Escolhendo-se $\alpha=-b / 3$ o termo quadrático é eliminado da equação. Com essa escolha os outros termos constantes podem ser rearranjados na forma,

$$
\begin{gathered}
c+3 \alpha^{2}+2 b \alpha=c+\frac{b^{2}}{3}-2 \frac{b^{2}}{3}=c-\frac{b^{2}}{3} \\
\alpha^{3}+b \alpha^{2}+c \alpha+d=-\frac{b^{3}}{27}+\frac{b^{3}}{9}-\frac{b c}{3}+d=\frac{2 b^{3}}{27}-\frac{b c}{3}+d
\end{gathered}
$$

Definindo,

$$
\begin{gathered}
p=c-\frac{b^{2}}{3} \\
q=\frac{2 b^{3}}{273}-\frac{b c}{}+d
\end{gathered}
$$

escreve-se,

$$
y^{3}+p y+q=0
$$

equação equivalente à equação completa do terceiro grau. A inexistência do termo na segunda potência em $y$ irá facilitar a solução da equação original.

A substituição do tipo $x=y-b / n$, em que $n$ é ordem da equação, é também válida para o segundo e quarto graus. Para o quarto grau o desenvolvimento é trabalhoso, mas para o segundo grau a demonstração vale a pena ser ilustrada nesse ponto. Se a equação do segundo grau é escrita na forma $x^{2}+b x+c=0$, pode-se tentar a substituição $x=y-b / 2$, para resultar em $y^{2}+b^{2} / 4+c=0$, ou $y= \pm \sqrt[2]{(b / 2)^{2}-c}$. A solução da equação do segundo grau é obtida somando-se $-b / 2$ ao valor de $y$. Portanto, a solução de $x^{2}+b x+c$ $=0$ é dada por, $x=-b / 2 \pm \sqrt[2]{(b / 2)^{2}-c}$ ou $y=\left(-b \pm \sqrt[2]{b^{2}-4 c}\right) / 2$, resultado certamente conhecido pelo leitor. 


\section{SOLUÇÃO DA EQUAÇÃO REDUZIDA}

A solução de $y^{3}+p y+q=0$ deve ser procurada, somando-se posteriormente o termo $-b / 3$, para que a solução de $x^{3}+b x^{2}+c x+d=0$ seja estabelecida, a exemplo do que foi feito para a equação do segundo grau. Procurando uma solução de (5) na forma, $y=u+v$ desenvolve-se,

$$
u^{3}+v^{3}+(3 u v+p)(u+v)+q=0
$$

Impondo a condição $3 u v+p=0$, implicando também que $v=-\frac{p}{3 u}$, obtém-se

$$
\left(u^{3}\right)^{2}+q u^{3}-\left(\frac{p}{3}\right)^{3}=0
$$

após multiplicação por $u^{3}$. A solução de (7), equação do segundo grau em $u^{3}$, será,

$$
u^{3}=-\frac{q}{2} \pm \sqrt{\left(\frac{q}{2}\right)^{2}+\left(\frac{p}{3}\right)^{3}}
$$

como $u^{3}+v^{3}=-q$ tem-se

$$
v^{3}=-\frac{q}{2} \mp \sqrt{\left(\frac{q}{2}\right)^{2}+\left(\frac{p}{3}\right)^{3}}
$$

Portanto, os dois valores distintos de $u^{3}$ e $v^{3}$ serão,

$$
\begin{aligned}
& u^{3}=-\frac{q}{2}+\sqrt{\left(\frac{q}{2}\right)^{2}+\left(\frac{p}{3}\right)^{3}} \\
& v^{3}=-\frac{q}{2}-\sqrt{\left(\frac{q}{2}\right)^{2}+\left(\frac{p}{3}\right)^{3}}
\end{aligned}
$$

\section{CONSTRUÇÃO DAS SOLUÇÕES}

Com os valores $u$ e $v$ pode-se construir a solução $y=u+v$ e, consequentemente, a solução da equação original. Os valores de $u$ e $v$ são obtidos extraindo-se a raiz cúbica de (10),

$$
\begin{gathered}
u_{1}=\sqrt[3]{-\frac{q}{2}+\sqrt[2]{\left(\frac{q}{2}\right)^{2}+\left(\frac{p}{3}\right)^{3}}} \quad v_{1}=\sqrt[3]{-\frac{q}{2}-\sqrt[2]{\left(\frac{q}{2}\right)^{2}+\left(\frac{p}{3}\right)^{3}}} \\
u_{2}=e^{2 \pi i / 3} \sqrt[3]{-\frac{q}{2}+\sqrt[2]{\left(\frac{q}{2}\right)^{2}+\left(\frac{p}{3}\right)^{3}}} \quad v_{2}=e \sqrt[3 \pi / 3]{-\frac{q}{2}-\sqrt[2]{\left(\frac{q}{2}\right)^{2}+\left(\frac{p}{3}\right)^{3}}} \\
u_{3}=e^{4 \pi i / 3} \sqrt[3]{-\frac{q}{2}+\sqrt[2]{\left(\frac{q}{2}\right)^{2}+\left(\frac{p}{3}\right)^{3}}} \quad v_{3}=e \sqrt[4 \pi i / 3]{-\frac{q}{2}-\sqrt[2]{\left(\frac{q}{2}\right)^{2}+\left(\frac{p}{3}\right)^{3}}}
\end{gathered}
$$

O leitor deve se lembrar de que a raiz cúbica de 1 , solução de $z^{3}=1$, tem as três raízes: $1, e^{2 \pi i / 3}, e^{4 \pi i / 3}$. Qualquer uma dessas soluções fornecerá $z^{3}=1$. Portanto, nove raízes são possíveis, $y_{i j} \equiv u_{i}+v_{j}$, mas somente três dessas funções serão soluções de (5). A solução da equação completa, equação (1) é obtida somando-se $-b / 3$ ao resultado obtido.

O problema está, de certa forma, resolvido, pois basta que as nove combinações possíveis sejam feitas e testadas. Entretanto, apesar de correta, essa maneira de resolver o problema pode ser muito simplificada se a álgebra das soluções é desenvolvida mais um pouco.
Observe que o procedimento de testar todas as soluções é trabalhoso e requer que número complexos sejam usados.

\section{SOLUÇÕES REAIS}

A procura das possíveis soluções pode ser simplificada se o problema é reduzido a soluções reais, caso que será de interesse em aplicações em físico-química. Para que o desenvolvimento das possíveis soluções reais fique mais claro é conveniente fazer as substituições, $e^{2 \pi i / 3}=-1 / 2+\mathrm{i} \sqrt{3} / 2$ e $e^{4 \pi i / 3}=-1 / 2-\mathrm{i} \sqrt{3} / 2$, transformando (11) em,

$$
\begin{aligned}
& u_{1}=\sqrt[3]{-\frac{q}{2}+\sqrt[2]{\left(\frac{q}{2}\right)^{2}+\left(\frac{p}{3}\right)^{3}}} \\
& u_{2}=-\frac{1}{2} \sqrt[3]{-\frac{q}{2}+\sqrt[2]{\left(\frac{q}{2}\right)^{2}+\left(\frac{p}{3}\right)^{3}}}+i \frac{\sqrt{3}}{2} \sqrt[3]{-\frac{q}{2}+\sqrt[2]{\left(\frac{q}{2}\right)^{2}+\left(\frac{p}{3}\right)^{3}}} \\
& u_{3}=-\frac{1}{2} \sqrt[3]{-\frac{q}{2}+\sqrt[2]{\left(\frac{q}{2}\right)^{2}+\left(\frac{p}{3}\right)^{3}}}-i \frac{\sqrt{3}}{2} \sqrt[3]{-\frac{q}{2}+\sqrt[2]{\left(\frac{q}{2}\right)^{2}+\left(\frac{p}{3}\right)^{3}}} \\
& v_{1}=\sqrt[3]{-\frac{q}{2}-\sqrt[2]{\left(\frac{q}{2}\right)^{2}+\left(\frac{p}{3}\right)^{3}}} \\
& v_{2}=-\frac{1}{2} \sqrt[3]{-\frac{q}{2}-\sqrt[2]{\left(\frac{q}{2}\right)^{2}+\left(\frac{p}{3}\right)^{3}}}+i \frac{\sqrt{3}}{2} \sqrt[3]{-\frac{q}{2}-\sqrt[2]{\left(\frac{q}{2}\right)^{2}+\left(\frac{p}{3}\right)^{3}}} \\
& v_{3}=-\frac{1}{2} \sqrt[3]{-\frac{q}{2}-\sqrt[2]{\left(\frac{q}{2}\right)^{2}+\left(\frac{p}{3}\right)^{3}}}-i \frac{\sqrt{3}}{2} \sqrt[3]{-\frac{q}{2}-\sqrt[2]{\left(\frac{q}{2}\right)^{2}+\left(\frac{p}{3}\right)^{3}}}
\end{aligned}
$$

O termo

$$
D=\left(\frac{q}{2}\right)^{2}+\left(\frac{p}{3}\right)^{3}
$$

que aparece em (12) irá determinar se as somas de $u$ e $v$ serão reais ou complexas. Três situações são possíveis, $D<0, D=0$ e $D>0$, e cada caso deve ser analisado separadamente.

Quando $D<0$ o termo $\sqrt{ } D$ irá contribuir com um fator complexo. Escrevendo a raiz cúbica na forma $z_{1}+i z_{2}$, desenvolve-se,

$$
\begin{array}{cc}
u_{1}=z_{1}+i z_{2} & v_{1}=z_{1}-i z_{2} \\
u_{2}=-\frac{1}{2}\left(z_{1}+i z_{2}\right)+i \frac{\sqrt{3}}{2}\left(z_{1}+i z_{2}\right) & v_{2}=-\frac{1}{2}\left(z_{1}-i z_{2}\right)+i \frac{\sqrt{3}}{2}\left(z_{1}-i z_{2}\right) \\
u_{3}=-\frac{1}{2}\left(z_{1}+i z_{2}\right)-i \frac{\sqrt{3}}{2}\left(z_{1}+i z_{2}\right) & v_{3}=-\frac{1}{2}\left(z_{1}-i z_{2}\right)-i \frac{\sqrt{3}}{2}\left(z_{1}-i z_{2}\right)
\end{array}
$$

Observe que não é necessário saber o valor explícito de $z_{1}$ e $z_{2}$. Essas são quantidades utilizadas unicamente na análise do tipo de solução. Depois dessa análise voltar-se-á a definição (11).

Quais as combinações possíveis de $u$ e $v$ em (14) que darão raízes reais? Uma das soluções será certamente $u_{1}+v_{1}$, pois nesse caso o termo complexo irá se cancelar. As outras duas soluções podem ser procuradas por simples inspeção também. Uma dessas soluções será $u_{2}+v_{3}$, pois,

$$
\begin{gathered}
u_{2}+v_{3}=-\frac{1}{2}\left(z_{1}+i z_{2}\right)+i \frac{\sqrt{3}}{2}\left(z_{1}+i z_{2}\right)-\frac{1}{2}\left(z_{1}-i z_{2}\right)-i \frac{\sqrt{3}}{2}\left(z_{1}-i z_{2}\right) \\
=-z_{1}-\frac{\sqrt{3}}{2} z_{2}
\end{gathered}
$$

A outra solução real será dada por, 


$$
\begin{gathered}
u_{3}+v_{2}=-\frac{1}{2}\left(z_{1}+i z_{2}\right)-i \frac{\sqrt{3}}{2}\left(z_{1}+i z_{2}\right)-\frac{1}{2}\left(z_{1}-i z_{2}\right)+i \frac{\sqrt{3}}{2}\left(z_{1}-i z_{2}\right) \\
=-z_{1}+\frac{\sqrt{3}}{2} z_{2}
\end{gathered}
$$

Apesar de trabalhosa, a prova de que as outras soluções são complexas é fácil de ser estabelecida.

Na condição em que $D=0$ desenvolve-se para (11),

$$
\begin{array}{cc}
u_{1}=\left(-\frac{q}{2}\right)^{1 / 3} & v_{1}=\left(-\frac{q}{2}\right)^{1 / 3} \\
u_{2}=-\frac{1}{2}\left(-\frac{q}{2}\right)^{1 / 3}+i \frac{\sqrt{3}}{2}\left(-\frac{q}{2}\right)^{1 / 3} & v_{2}=-\frac{1}{2}\left(-\frac{q}{2}\right)^{1 / 3}+i \frac{\sqrt{3}}{2}\left(-\frac{q}{2}\right)^{1 / 3} \\
u_{3}=-\frac{1}{2}\left(-\frac{q}{2}\right)^{1 / 3}-i \frac{\sqrt{3}}{2}\left(-\frac{q}{2}\right)^{1 / 3} & v_{3}=-\frac{1}{2}\left(-\frac{q}{2}\right)^{1 / 3}-i \frac{\sqrt{3}}{2}\left(-\frac{q}{2}\right)^{1 / 3}
\end{array}
$$

As soluções reais serão dada por: $u_{1}+v_{1}, u_{2}+v_{3}$ e $u_{3}+v_{2}$. Observe que $u_{2}+v_{3}=u_{3}+v_{2}=-2\left(u_{1}+v_{1}\right)$. Se adicionalmente $q=0$ as soluções de (5) serão iguais a zero e, nesse caso, a solução da equação original será $x=-b / 3$. Com $\mathrm{D}>0$ observa-se, por (11), que a única solução real corresponderá a $u_{1}+v_{1}$. Portanto, as possíveis soluções reais de $y^{3}+p y+q=0$ serão: a) $u_{1}+v_{1}, u_{2}+v_{3}$ e $u_{3}+v_{2}$; b) $D=0, u_{1}+v_{1}$ e $u_{2}+v_{3}=u_{3}+v_{2}$; e c) $D>0, u_{1}+v_{1}$.

Para o caso em que $D<0$, uma forma prática, em termos de funções trigonométricas pode ser utilizada,

$$
\begin{gathered}
x_{3}=2 \sqrt[3]{r} \cos \left(\frac{\phi}{3}\right)-\frac{b}{3} \\
x_{2}=2 \sqrt[3]{r} \cos \left(\frac{\phi+2 \pi}{3}\right)-\frac{b}{3} \\
x_{1}=2 \sqrt[3]{r} \cos \left(\frac{\phi+4 \pi}{3}\right)-\frac{b}{3}
\end{gathered}
$$

com, $\quad r=\sqrt[2]{-p^{3} / 27}$ e $\phi=\operatorname{acos}(-q / 2 r)$. Esse resultado é provado usando-se a a forma $e^{i \theta}=\cos \theta+i \operatorname{sen} \theta$ nas soluções reais. A Tabela 1 resume a análise das possíveis soluções da equação completa do terceiro grau. Somente soluções reais são apresentadas.

Tabela 1. Possíveis soluções reais de $x^{3}+b x^{2}+c x=d=0$

\begin{tabular}{c}
\hline Sinal de $D$ \\
\hline$x_{3}=2 \sqrt[3]{r} \cos \left(\frac{\varphi}{3}\right)-\frac{b}{3}$ \\
$x_{2}=2 \sqrt[3]{r} \cos \left(\frac{\varphi+2 \pi}{3}\right)-\frac{b}{3}$ \\
$x_{1}=2 \sqrt[3]{r} \cos \left(\frac{\varphi+4 \pi}{3}\right)-\frac{b}{3}$ \\
$x_{3}=2\left(-\frac{q}{2}\right)^{1 / 3}-\frac{b}{3}$ \\
$x_{2}=x_{1}=-\left(-\frac{q}{2}\right)^{1 / 3}-\frac{b}{3}$ \\
$D>0 \quad \sqrt[3]{-\frac{q}{2}+\sqrt[2]{\left(\frac{q}{2}\right)^{2}+\left(\frac{p}{3}\right)^{3}}}+\sqrt[3]{-\frac{q}{2}-\sqrt[2]{\left(\frac{q}{2}\right)^{2}+\left(\frac{p}{3}\right)^{3}}}-\frac{b}{3}$ \\
\hline
\end{tabular}

Comoum primeiro exemplo considere a equação, $x^{3}-6 x^{2}+11 x-6=0$, que admite as soluções 1,2 e 3 . Portanto, $b=-6, c=11, d=-6$, $p=c-b^{2} / 3=-1$ e $q=2 b^{3} / 27-b c / 3+d=0$, acarretando D negativo.
Aplicando o resultado da Tabela 1 obtém-se

$$
\begin{aligned}
& r=\sqrt[2]{-\frac{p^{3}}{27}}=0,1925 \\
& \phi=\operatorname{acos}\left(-\frac{q}{2 r}\right)=\frac{\pi}{2}
\end{aligned}
$$

A substituição na solução trigonométrica fornece as soluções exatas. Como outro exemplo pode-se tentar, $x^{3}-24 x^{2}+191 x-504=0$, que terá soluções $x=(7,8,9)$.

\section{EXEMPLOS TRABALHADOS POR CARDANO}

Na obra de G. Cardano ${ }^{1}$ são fornecidos inúmeros exemplos de solução de equações cúbicas. O assunto é tratado do capítulo XI até o XXV. O início do livro relata uma introdução ao assunto e do capítulo XXV ao XL são abordadas equações de quarto grau. As soluções envolvendo números complexos não eram fornecidas por Cardano, pois considerava esse tipo de resultado como artificial. Com um raciocínio geométrico Cardano evitava colocar resultados negativos, apesar de aceitar algumas soluções de equações quadráticas. Por exemplo, na p. 10, mostra que 3 e -3 são soluções de $x^{2}=9$. Na verdade, o trabalho de Cardano provocou o estudo de números complexos, que iria aparecer de forma formal em 1572, como discutido na referência 3. Desprezar as soluções negativas e complexas não é absurdo, se o interesse está nas aplicações. Por exemplo, no cálculo do volume na equação de van der Waals ${ }^{4}$ ou no cálculo de concentrações em equilíbrio químico, as soluções apresentadas por Cardano são as que fazem sentido. A prova da solução, como em (11), foi dada no capítulo XI, regra I, mas não nessa forma matemática explícita, mas com uma apresentação textual. A forma dissertativa era uma característica do trabalho de Cardano. Por exemplo, o título do capítulo XI é Sobre o cubo e a primeira potência sendo igual a um número, o que poderia ter sido escrito como, $x^{3}+a x=b$.

Os resultados aqui apresentados, pela combinação das soluções (11) ou pelos apresentados na Tabela 1, serão comparados com alguns exemplos elaborados por Cardano. ${ }^{1} \mathrm{Na}$ p. 20 a solução de $x^{3}+4=3 x^{2}+5 x$ é desejada. O resultado numérico calculado vale, 4 , 0,6180 e -1,6180. Numa forma elegante Cardano coloca a solução na forma, $4, \sqrt{1 \frac{1}{4}}+\frac{1}{2} \mathrm{e}-\left(\sqrt{1 \frac{1}{4}}+\frac{1}{2}\right)$, equivalendo ao resultado numérico. A solução negativa foi chamada de falsa por Cardano.

Na p. 124 fica evidente que Cardano evita as soluções envolvendo raiz de números negativos, ao procurar a solução de $x^{3}+6 x^{2}+12 x=22$, fornecendo somente a solução real 1,107 . As soluções complexas conjugadas, $(-3,553+i 2,6910)$ e $(-3,553-i 2,6910)$ não são mencionadas

\section{CÁLCULO DO VOLUME NA EQUAÇÃO DE van der WAALS}

A equação de van der Waals, $\left(p+a / \bar{V}^{2}\right)(\bar{V}-b)$, em que $\bar{V}$ se refere ao volume molar, pode ser escrita na forma equivalente,

$$
\bar{V}^{3}-\left(b+\frac{R T}{p}\right) \bar{V}^{2}+\frac{a}{p} \bar{V}-\frac{a b}{p}=0
$$

A solução de uma equação completa do terceiro grau é necessária para que o volume seja estabelecido num sistema descrito pela

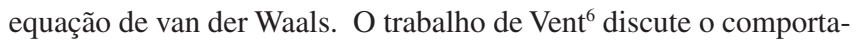
mento dos gases previsto pela equação de estado de van der Waals, usando métodos numéricos para o cálculo do volume molar. Deiters ${ }^{7}$ 
desenvolve um algoritmo que explora a combinação da fórmula de Cardano com métodos iterativos. Entretanto, como mostrado neste trabalho, a solução exata pode ser encontrada.

A molécula de $\mathrm{CO}_{2}$ será usada como protótipo para exemplificar o cálculo do volume pelo método de Cardano. Para esse sistema tem-se $a=3,592 \mathrm{~atm} \mathrm{~L}^{2} \mathrm{~mol}^{-2}, \mathrm{~b}=$ $0,04267 \mathrm{~L} \mathrm{~mol}^{-1}$ e $T_{c}=304 \mathrm{~K}^{8}$ Porém, pode-se aprender sobre as possíveis soluções do volume, discutindo-se algumas isotermas, como apresentado na Figura 1 para $T=280 \mathrm{~K}$, $T=T_{c}=304 \mathrm{Ke} T_{c}=304 \mathrm{~K}$. Se $p=50 \mathrm{~atm}$ em $T=280 \mathrm{~K}$ observa-se que a solução de (19) apresenta três soluções reais. Se o sistema se encontra no estado crítico $\left(p_{c}, V_{c}, T_{c}\right)$, somente uma solução será encontrada. Para qualquer outra pressão acima da temperatura crítica somente uma raiz real pode acontecer. Esse é o caso também quando $T=310 \mathrm{~K}$. Portanto, a Figura 1 ilustra também os três casos possíveis em relação ao valor de $D$ : a) $D$ menor do que zero $\left(T<T_{c}\right)$; b) $D$ igual a $\operatorname{zero}\left(T=T_{c}\right)$ e c) $D$ maior do que zero $\left(T>T_{c}\right)$, com respectivas soluções de acordo com a Tabela 1 .

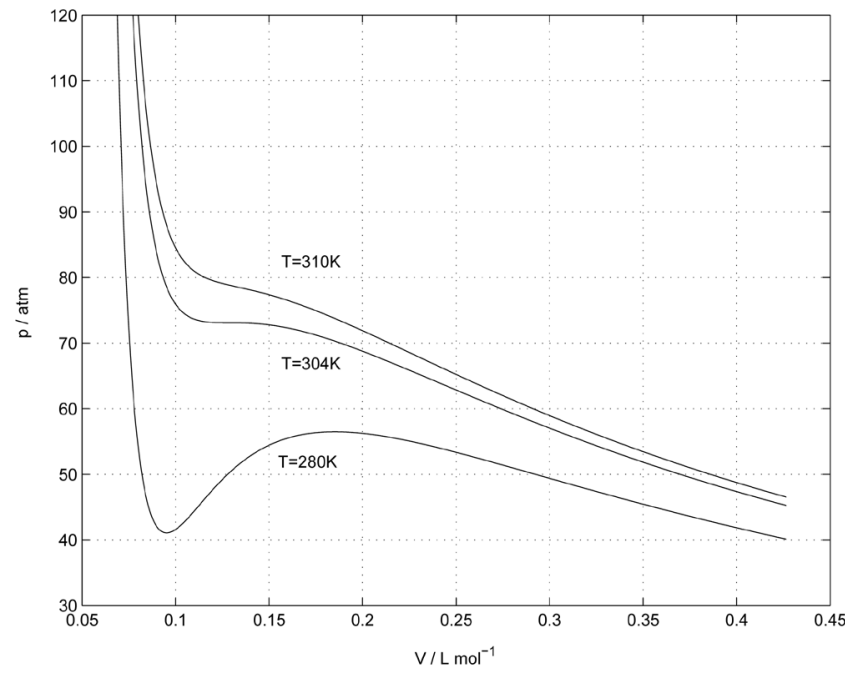

Figura 1. Isoterma de van der Waals para a molécula de $\mathrm{CO}_{2}$

Observe que algumas quantidades na equação de van der Waals, como a constante $b$ ou a pressão $p$, se confundem com a notação usada normalmente na solução das equações algébricas. Portanto, uma das duas notações deve ser modificada. Usando b', c', d',p', q' para representar as constantes da equação do terceiro grau calcula-se para o sistema de van der Waals,

$$
\begin{gathered}
b^{\prime}=-\left(b+\frac{R T}{p}\right) \\
c^{\prime}=\frac{a}{p} \\
d^{\prime}=-\frac{a b}{p} \\
p^{\prime}=c^{\prime}-\frac{b^{\prime 2}}{3}=\frac{a}{p}-\frac{\left(b+\frac{R T}{p}\right)^{2}}{3} \\
q^{\prime}=\frac{2 b^{\prime 3}}{27}-\frac{b^{\prime} c^{\prime}}{3}+d^{\prime}=-\frac{2\left(b+\frac{R T}{p}\right)^{3}}{27}+\frac{\left(b+\frac{R T}{p}\right) \frac{a}{p}}{3}-\frac{a b}{p}
\end{gathered}
$$

Para $T=280 \mathrm{~K}$ e $p=50 \mathrm{~atm} \operatorname{com} R=0,08206 \mathrm{~atm} \mathrm{~L} \mathrm{~mol}^{-1} \mathrm{~K}^{-1}$ calcula-se para o $\mathrm{CO}_{2}$,

$$
\begin{gathered}
b^{\prime}=-0,3995 \\
c^{\prime}=0,0521 \\
d^{\prime}=-0,0022 \\
p^{\prime}=-0,0123 \\
q^{\prime}=-4,6270 \times 10^{-4}
\end{gathered}
$$

acarretando em $D<0$, como deveria ser. A substituição desses valores na solução estabelecida, forma trigonométrica, fornece $V_{1}=0,2936 \mathrm{~L}$, $V_{2}=0,1221 \mathrm{~L}$ e $V_{2}=0,0865 \mathrm{~L}$ para as três raízes. Pode-se constatar pela Figura 1 que esse é o resultado esperado.

Como uma consequência direta da aplicação da solução de Cardano, obtida para o volume em van der Waals, fica-se motivado a prosseguir com outras aplicações. Uma delas se refere ao cálculo do fator de compressibilidade, $Z=V / V_{\text {ideal }}$, tomando a solução real de maior valor. $\mathrm{O}$ cálculo para $T=310 \mathrm{~K}$ dessa quantidade apresenta-se na Figura 2. O fator de compressibilidade para outras temperaturas é calculado de forma semelhante.

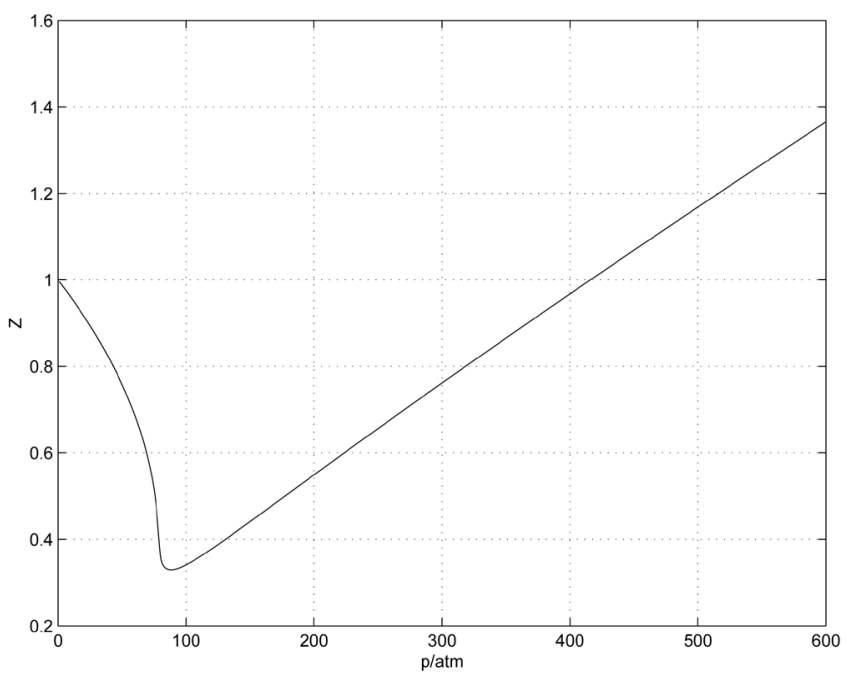

Figura 2. Fator de compressiblidade de van der Waals para a molécula de $\mathrm{CO}_{2} \mathrm{em} \mathrm{T}=310 \mathrm{~K}$

\section{FATOR DE COMPRESSIBILIDADE ANALÍTICO}

O fator de compressibilidade pode ainda ser colocado numa forma analítica, pois todos os parâmetros para o cálculo são conhecidos. Para ilustrar, considera-se a temperatura maior do que a temperatura crítica. Pelos valores das constantes da solução algébrica, como em (20), pode-se usar a solução resumida na Tabela 1 para se estabelecer,

$$
\begin{gathered}
\bar{V}=\sqrt[3]{\frac{(p b+R T)^{3}}{27 p^{3}}-\frac{(p b+R T) a}{6 p^{2}}+\frac{a b}{2 p}+\sqrt[2]{\left(\frac{(p b+R T)^{3}}{27 p^{3}}-\frac{(p b+R T) a}{6 p^{2}}+\frac{a b}{2 p}\right)^{2}+\left(\frac{a}{p}-\frac{(p b+R T)^{2}}{3 p^{2}}\right)^{3}}} \\
+\sqrt[3]{\frac{(p b+R T)^{3}}{27 p^{3}}-\frac{(p b+R T) a}{6 p^{2}}+\frac{a b}{2 p}-\sqrt[2]{\left(\frac{(p b+R T)^{3}}{27 p^{3}}-\frac{(p b+R T) a}{6 p^{2}}+\frac{a b}{2 p}\right)^{2}+\left(\frac{a}{p}-\frac{(p b+R T)^{2}}{3 p^{2}}\right)^{3}}} \\
+\frac{(p b+R T)}{3 p}
\end{gathered}
$$

Investigou-se a possibilidade de mais simplificações nessa expressão, mas isso não será vantajoso, pois teria que se desenvolver os termos ao quadrado e ao cubo. A expressão iria ficar mais complicada, perdendo a simetria. $\mathrm{O}$ fator de compressibilidade é obtido dividindo-se esse valor por $R T / p$. O resultado é o mesmo apresentado na Figura 2. Isso mostra que (22) pode ser muito útil, não somente para cálculos numéricos, mas oferecendo uma alternativa para o ensino do fator de compressibilidade em sistema descritos pela equação de van der Waals. 


\section{LEI DAS ÁREAS IGUAIS DE MAXWELL}

Se um ciclo isotérmico, formado por uma pressão constante na qual há formação de líquidos e a isoterma de van der Waals é considerada tem-se, $T \oint d S=\oint d W$, ou ainda $\oint d W=0$. O trabalho total no ciclo isotérmico é zero, tendo como consequência a lei das áreas iguais de Maxwell. ${ }^{9}$ A diferença entre essas duas áreas pode ser obtida desenvolvendo-se,

$$
\oint d W=\int_{\bar{V}_{1}}^{\bar{V}_{3}} p d \bar{V}-p^{*}\left(\bar{V}_{3}-\bar{V}_{1}\right)
$$

em que $p$ satisfaz a equação de van der Waals e $p^{*}$ equivale à pressão para a qual os três volumes são calculados. Observe que para a aplicação da lei das áreas iguais de Maxwell tem de se ter $D<0$. O trabalho no ciclo pode ser desenvolvido,

$\oint d W=R T \ln \left(\frac{\bar{V}_{3}-b}{\bar{V}_{1}-b}\right)+a\left(\frac{11}{\bar{V}_{3}}-\overline{\bar{V}}_{1}\right)-p^{*}\left(\bar{V}_{3}-\bar{V}_{1}\right)$

Então, para um dado $p^{*}$ os volumes pela solução analítica podem ser usados para o cálculo de $\mathrm{o} d W$. A pressão na qual o trabalho no ciclo mudar de sinal deve ser tomada como a linha de amarração no equilíbrio líquido-vapor.

A Tabela 2 mostra a variação da pressão, cálculo dos volumes (somente os extremos) e a diferença de área. Observa-se que a pressão de $52 \mathrm{~atm}$ corresponde ao ponto em que o trabalho se torna zero, estabelecendo assim a pressão em que haverá formação do líquido.

Tabela 2. Aplicação das leis das áreas iguais de Maxwell. Unidades em atm e L

\begin{tabular}{lccc}
\hline $\mathrm{p}$ & $\bar{V}_{1}$ & $\bar{V}_{3}$ & $\oint_{d W}$ \\
\hline 50 & 0,08200 & 0,2923 & 0,43 \\
51 & 0,08147 & 0,2800 & 0,23 \\
52 & 0,08098 & 0,2675 & 0,00 \\
53 & 0,08053 & 0,2546 & $-0,15$ \\
54 & 0,08010 & 0,2410 & $-0,32$ \\
55 & 0,07970 & 0,2260 & $-0,47$ \\
\hline
\end{tabular}

\section{CONCLUSÃO}

O trabalho de Cardano sobre soluções analíticas de equações do terceiro grau foi apresentado de forma didática com aplicações no cálculo do volume na equação de estado de van der Waals. Três exemplos são fornecidos inicialmente para ilustar a simplicidade do método. Alguns exemplos trabalhados por Cardano são também apresentados.

O cálculo do volume na equação de van der Waals foi feito na região abaixo e acima da temperatura crítica, tomando a molécula de $\mathrm{CO}_{2}$ como protótipo. Desses volumes obtidos e para temperatura acima da crítica calculou-se o fator de compressibilidade. Como um resultado da procura por soluções analíticas para o volume foi possível estabelecer uma expressão analítica para o fator de compressibilidade.

Como outra aplicação do método de Cardano usou-se a solução estabelecida para o volume para se calcular a condição em que $\oint d W=0$. Isso permitiu, de forma simples, que a pressão para a transição líquido-vapor na temperatura especificada pudesse ser estabelecida. O raciocínio pode ser continuado para o estabelecimento da curva de coexistência líquido-vapor.

As expressões estabelecidas por Cardano e usadas no presente trabalho são extremamente facéis de serem utilizadas, envolvendo somente substituições elementares. Isso indica que o método de Cardano pode ser usado em cursos introdutórios de físico-química, tanto no cálculo numérico ou na solução analítica. Por exemplo, o fator de compressibilidade obtido de forma analítica pode ser usado para se estabelecer a curva do fator de compressibilidade em função da pressão, resultado normalmente apresentado somente na forma gráfica.

\section{AGRADECIMENTOS}

J. P. Braga gostaria de agradecer ao CNPq pela ajuda financeira.

\section{REFERÊNCIAS}

1. Cardano, G.; Ars Magna, The Rules of Algebra, Dover Publications: New York, 1968.

2. Boyer, C. B.; História da Matemática, Ed. Edgard Blucher: São Paulo, 1981.

3. Struik, D. J.; A source book in mathematics, 1200-1800, Harvard University Press: Cambridge, 1969.

4. Braga, J. P.; Fundamentos de Físico-Química, Edi. UFV: Viçosa, 2004.

5. Smirnov, V.; Cours de Mathématiques supérieuers, Ed. Mir: Moscou, 1969, vol. 1 .

6. Vent, S. Le; International Journal of Mechanical Engineering Education 2001, 29, 257.

7. Deiters, U. K.; AIChE J. 2002, 48, 882.

8. Lide, D. R.; Handbook of Chemistry and Physics, CRC Press: Florida, 2004.

9. Fermi, E.; Thermodynamics, Dover Publications: New York, 1956. 